\title{
GENERALIZED D. H. LEHMER PROBLEM OVER SHORT INTERVALS
}

\author{
PING XI \\ School of Science, Xi'an Jiaotong University, Xi'an 710049, P. R. China \\ email: xprime@163.com \\ and YUAN YI \\ School of Science, Xi'an Jiaotong University, Xi'an 710049, P. R. China and Department of Mathematics, \\ The University of Iowa, Iowa City, IA 52242-1419, USA \\ email:yuanyi@mail.xjtu.edu.cn
}

(Received 21 December 2009; accepted 1 July 2010; first published online 13 December 2010)

Abstract. Let $n \geqslant 2$ be a fixed positive integer, $q \geqslant 3$ and $c, \ell$ be integers with $(n c, q)=1$ and $\ell \mid n$. Suppose $\mathcal{A}$ and $\mathcal{B}$ consist of consecutive integers which are coprime to $q$. We define the cardinality of a set:

$$
N(\mathcal{A}, \mathcal{B}, c, n, \ell ; q)=\#\{(a, b) \in \mathcal{A} \times \mathcal{B} \mid a b \equiv c(\bmod q),(a+b, n)=\ell\}
$$

The main purpose of this paper is to use the estimates of Gauss sums and Kloosterman sums to study the asymptotic properties of $N(\mathcal{A}, \mathcal{B}, c, n, \ell ; q)$, and to give an interesting asymptotic formula for it.

2010 Mathematics Subject Classification. Primary 11A07, 11N37; Secondary $11 \mathrm{~L} 05$.

1. Introduction. Let $q \geqslant 3$ be an integer. For each integer $a$ with $1 \leqslant a<$ $q,(a, q)=1$, there is a unique integer $b$ with $1 \leqslant b<q$ such that $a b \equiv 1(\bmod q)$. Let $N(q)$ denote the number of solutions of the congruence equation $a b \equiv 1(\bmod q)$ with $1 \leqslant a, b<q, 2 \nmid a+b$. That is

$$
N(q)=\#\{(a, b) \in[1, q] \times[1, q] \mid a b \equiv 1(\bmod q), 2 \nmid a+b\},
$$

where $\# \mathcal{S}$ denotes the cardinality of the set $\mathcal{S}$. Thus, $N(q)$ denotes the number of integers $a, 1 \leqslant a<q,(a, q)=1$, such that $a$ and its inverse $b(\bmod q)$ are of opposite parity.

For an odd prime $p$, D. H. Lehmer posed the problem to find $N(p)$ or at least to say something nontrivial about it (see Problem F12 of [2], p. 381). Wenpeng Zhang [8] has given an asymptotic estimate:

$$
N(p)=\frac{1}{2} p+O\left(p^{1 / 2} \log ^{2} p\right)
$$

Supported by N. S. F. (No.10601039) of P. R. China. 
Later, Wenpeng Zhang $[\mathbf{9 , 1 0}]$ also proved that for every odd integer $q \geqslant 3$,

$$
N(q)=\frac{1}{2} \varphi(q)+O\left(q^{1 / 2} \tau^{2}(q) \log ^{2} q\right),
$$

where $\varphi(q)$ is the Euler function and $\tau(q)$ is the divisor function.

The classical problem has been generalized by many scholars (see [5-7], et al.). Recently, Yaming Lu and Yuan Yi [3] studied a generalization of the D. H. Lehmer problem over short intervals. Let $n \geqslant 2$ be a fixed positive integer, $q \geqslant 3$ and $c$ be integers with $(n c, q)=1$. We define

$$
r_{n}\left(\theta_{1}, \theta_{2}, c ; q\right)=\#\left\{(a, b) \in\left[1, \theta_{1} q\right] \times\left[1, \theta_{2} q\right] \mid a b \equiv c(\bmod q), n \nmid a+b\right\},
$$

where $0<\theta_{1}, \theta_{2} \leqslant 1$. In [3], it is obtained that

$$
r_{n}\left(\theta_{1}, \theta_{2}, c ; q\right)=\left(1-\frac{1}{n}\right) \theta_{1} \theta_{2} \varphi(q)+O\left(q^{1 / 2} \tau^{6}(q) \log ^{2} q\right),
$$

where the $O$-constant depends only on $n$.

In this paper, we consider a more extensive generalization of the D. H. Lehmer problem over short intervals, which may be of great arithmetical interest.

Suppose $\mathcal{A}$ and $\mathcal{B}$ consist of consecutive integers which are coprime to $q$, that is,

$$
\begin{gathered}
\mathcal{A}=\{n \in \mathcal{Q}: M<n \leqslant M+A\}, \\
\mathcal{B}=\{n \in \mathcal{Q}: N<n \leqslant N+B\},
\end{gathered}
$$

where $M, N, A>0, B>0$ are integers, $\mathcal{Q}$ is a reduced residue system modulo $q$. Let $n \geqslant 2$ be a fixed positive integer, $q \geqslant 3$ and $c, \ell$ be integers with $(n c, q)=1$ and $\ell \mid n$, and define

$$
N(\mathcal{A}, \mathcal{B}, c, n, \ell ; q)=\#\{(a, b) \in \mathcal{A} \times \mathcal{B} \mid a b \equiv c(\bmod q),(a+b, n)=\ell\} .
$$

The main purpose of this paper is to use the estimates of Gauss sums and Kloosterman sums to study the asymptotic properties of $N(\mathcal{A}, \mathcal{B}, c, n, \ell ; q)$, and to give an interesting asymptotic formula for it. In fact, we have the following.

THEOREM 1. Let $n \geqslant 2$ be a fixed positive integer, $q \geqslant 3$ and $c, \ell$ be integers with $(n c, q)=1$ and $\ell \mid n$, the sets $\mathcal{A}$ and $\mathcal{B}$ are defined by (4) and (5). Then, as $q \rightarrow+\infty$, we have the asymptotic formula

$$
\begin{aligned}
N(\mathcal{A}, \mathcal{B}, c, n, \ell ; q)= & \frac{\# \mathcal{A} \# \mathcal{B}}{n} \varphi\left(\frac{n}{\ell}\right) \varphi^{-1}(q)+O\left(\sqrt{\frac{\# \mathcal{A} \# \mathcal{B}}{q}} \tau^{3}(q) \cdot n 2^{\omega(n / \ell)}\right) \\
& +O\left(q^{1 / 2} \tau^{3}(q) \log ^{2} q \cdot 2^{\omega(n / \ell)}\right),
\end{aligned}
$$

where $\varphi(n)$ is the Euler function, $\tau(q)$ is the divisor function, $\omega(q)$ denotes the number of distinct prime factors of $q$, \#A denotes the cardinality of $\mathcal{A}$ and two $O$-constants are both absolute.

We can see that the estimate is nontrivial when $\# \mathcal{A} \# \mathcal{B} \gg q^{3 / 2+\epsilon}$, where the implied constant depends at most on $n$ and $\epsilon$. 
2. Lemmas. In order to prove Theorem 1, we require the following lemmas. First, for integers $m, n, q$, we introduce the classical Kloosterman sum:

$$
S(m, n ; q)=\sum_{\substack{a \bmod q \\(a, q)=1}} e\left(\frac{m a+n \bar{a}}{q}\right),
$$

where $e(x)=e^{2 \pi i x}, a \bar{a} \equiv 1(\bmod q)$.

LEMMA 1. Let $m, n, q$ be integers, $q \geqslant 3$, then we have the upper bound

$$
|S(m, n ; q)| \leqslant q^{1 / 2}(m, n, q)^{1 / 2} \tau(q) .
$$

Proof. See [1].

Denote by $\chi$ a Dirichlet character $\bmod q$, by $\chi^{0}$ the principal one, and by $m$ an integer. The well known Gauss sum is defined by

$$
G(m, \chi)=\sum_{h \bmod q} \chi(h) e\left(\frac{m h}{q}\right) .
$$

We also require some properties of Gauss sums, which are stated as the following two lemmas.

LEMMA 2. For any positive integers $q$ and $m$, we have

$$
G\left(m, \chi^{0}\right)=\mu\left(\frac{q}{(m, q)}\right) \varphi(q) \varphi^{-1}\left(\frac{q}{(m, q)}\right),
$$

where $\mu(n)$ is the Möbius function.

Proof. See [4], Section 1.2, Lemma 2.

Lemma 3. Let $q$ and $c$ be two integers with $q \geqslant 3,(c, q)=1$. Then for any integers $a$ and $b$, we have

$$
\sum_{\chi \neq \chi^{0}} \chi(c) G(a, \chi) G(b, \chi) \ll \varphi(q) q^{1 / 2}(a, q)^{1 / 2}(b, q)^{1 / 2} \tau(q),
$$

where the $O$-constant is absolute.

Proof. By using Lemma 1, we can easily deduce that

$$
\begin{aligned}
\sum_{\chi \bmod q} \chi(c) G(a, \chi) G(b, \chi) & =\sum_{\chi \bmod q} \chi(c) \sum_{s=1}^{q} \chi(s) e\left(\frac{a s}{q}\right) \sum_{t=1}^{q} \chi(t) e\left(\frac{b t}{q}\right) \\
& =\sum_{s=1}^{q} \sum_{t=1}^{q} e\left(\frac{a s+b t}{q}\right) \sum_{\chi \bmod q} \chi(s t c) \\
& =\varphi(q) \sum_{s=1}^{q} \sum_{t=1}^{q} e\left(\frac{a s+b t}{q}\right) \\
& =\varphi(q) S(a, b \bar{c} ; q) \\
& \ll \varphi(q) q^{1 / 2}(a, b, q)^{1 / 2} \tau(q) .
\end{aligned}
$$


On the other hand, Lemma 2 indicates that

$$
\begin{aligned}
G\left(a, \chi^{0}\right) G\left(b, \chi^{0}\right) & =\mu\left(\frac{q}{(a, q)}\right) \mu\left(\frac{q}{(b, q)}\right) \varphi^{2}(q) \varphi^{-1}\left(\frac{q}{(a, q)}\right) \varphi^{-1}\left(\frac{q}{(b, q)}\right) \\
& \ll \varphi^{2}(q) \frac{(a, q)(b, q)}{q^{2}} \tau\left(\frac{q}{(a, q)}\right) \tau\left(\frac{q}{(b, q)}\right) \\
& \ll \varphi^{2}(q) \frac{(a, q)(b, q)}{q^{2}} \frac{q}{\sqrt{(a, q)(b, q)}} \\
& \ll \varphi(q)(a, q)^{1 / 2}(b, q)^{1 / 2} .
\end{aligned}
$$

Then Lemma 3 follows from (6) and (7) immediately.

Note: A slight weaker estimate than Lemma 3 can be found in [3].

The following two lemmas focus on the estimation for exponential sums.

LemMa 4. Let $N$ be a positive integer, $\alpha$ be a real number. Then we have

$$
\left|\sum_{n \leqslant N} e(\alpha n)\right| \leqslant \min \left(N, \frac{1}{2\|\alpha\|}\right),
$$

where $\|x\|=\min _{n \in \mathbb{Z}}|x-n|$.

Proof. The estimate is well known, the proof can be found in [4], Section 5.1.

LeMma 5. Assume that $U$ is a positive real number, $K_{0}$ an integer, $K$ a positive integer, $\alpha$ and $\beta$ two arbitrary real numbers. If $\alpha$ can be written in the form

$$
\alpha=\frac{h}{q}+\frac{\theta}{q^{2}} \quad(q, h)=1, \quad q \geqslant 1, \quad|\theta| \leqslant 1,
$$

we have

$$
\sum_{k=K_{0}+1}^{K_{0}+K} \min \left(U, \frac{1}{\|\alpha k+\beta\|}\right) \ll\left(\frac{K}{q}+1\right)(U+q \log q),
$$

where the implied constant is absolute.

Proof. See reference [4], Section 5.1, Lemma 3.

3. Proof of Theorem 1. In this section, we shall complete the proof of Theorem 1. From the orthogonality relation for Dirichlet characters modulo $q$, one can obtain that

$$
\begin{aligned}
& N(\mathcal{A}, \mathcal{B}, c, n, \ell ; q)=\frac{1}{\varphi(q)} \sum_{\chi \bmod } \sum_{\substack{a \in \mathcal{A} \\
(a+b, n)=\ell}} \sum_{b \in \mathcal{B}} \chi(a b) \bar{\chi}(c)
\end{aligned}
$$

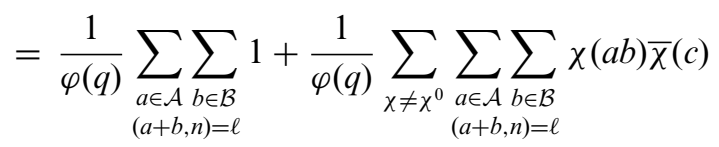

$$
\begin{aligned}
& :=I_{1}+I_{2} \text {. }
\end{aligned}
$$


We shall estimate $I_{1}$ and $I_{2}$ respectively. Firstly,

$$
\begin{aligned}
I_{1} & =\frac{1}{\varphi(q)} \sum_{\substack{a \in \mathcal{A} \\
(a+b, n)=\ell}} \sum_{b \in \mathcal{B}} 1=\frac{1}{\varphi(q)} \sum_{\substack{a \in \mathcal{A} \\
\ell \mid a+b}} \sum_{b \in \mathcal{B}} \sum_{r \mid\left(\frac{a+b}{\ell}, \frac{n}{\ell}\right)} \mu(r) \\
& =\frac{1}{\varphi(q)} \sum_{a \in \mathcal{A}} \sum_{r \mid \frac{n}{\ell}} \mu(r) \sum_{\substack{b \in \mathcal{B} \\
b \equiv-a(\bmod r \ell)}} 1 \\
& =\frac{1}{\varphi(q)} \sum_{a \in \mathcal{A}} \sum_{r \mid \frac{n}{\ell}} \mu(r)\left(\frac{\# \mathcal{B}}{r \ell}+O(1)\right) \\
& =\frac{\# \mathcal{B}}{\varphi(q) \ell} \sum_{a \in \mathcal{A}} \sum_{r \mid \frac{n}{\ell}} \frac{\mu(r)}{r}+O\left(2^{\omega(n / \ell)}\right) \\
& =\frac{\# \mathcal{A} \# \mathcal{B}}{n} \varphi\left(\frac{n}{\ell}\right) \varphi^{-1}(q)+O\left(2^{\omega(n / \ell)}\right) .
\end{aligned}
$$

Secondly,

$$
\begin{aligned}
I_{2} & =\frac{1}{\varphi(q)} \sum_{\chi \neq \chi^{0}} \bar{\chi}(c) \sum_{\substack{a \in \mathcal{A} \\
(a+b, n)=\ell}} \chi(a b)=\frac{1}{\varphi(q)} \sum_{\chi \neq \chi^{0}} \bar{\chi}(c) \sum_{r \mid \frac{n}{\ell}} \mu(r) \sum_{\substack{a \in \mathcal{A} \\
r \mid \frac{a+b}{\ell}}} \chi(a b) \\
& =\frac{1}{\varphi(q) \ell} \sum_{\chi \neq \chi^{0}} \bar{\chi}(c) \sum_{r \mid \frac{n}{\ell}} \frac{\mu(r)}{r} \sum_{m \leqslant r \ell} \sum_{a \in \mathcal{A}} \sum_{b \in \mathcal{B}} e\left(\frac{m(a+b)}{r \ell}\right) \chi(a b) \\
& =\frac{1}{\varphi(q) \ell} \sum_{\chi \neq \chi^{0}} \bar{\chi}(c) \sum_{r \mid \frac{n}{\ell}} \frac{\mu(r)}{r} \sum_{m \leqslant r \ell} \sum_{a \in \mathcal{A}} \chi(a) e\left(\frac{m a}{r \ell}\right) \sum_{b \in \mathcal{B}} \chi(b) e\left(\frac{m b}{r \ell}\right) .
\end{aligned}
$$

Note that for any non-principal character $\chi \bmod q$,

$$
\chi(a)=\frac{1}{q} \sum_{s \leqslant q} G(s, \chi) e\left(-\frac{a s}{q}\right)
$$

thus,

$$
\sum_{a \in \mathcal{A}} \chi(a) e\left(\frac{m a}{r \ell}\right)=\frac{1}{q} \sum_{s \leqslant q} G(s, \chi) \sum_{a \in \mathcal{A}} e\left(\left(\frac{m}{r \ell}-\frac{s}{q}\right) a\right) .
$$

Combining (10) and (11), and making use of Lemma 3 and 4, we have

$$
\begin{aligned}
I_{2}= & \frac{1}{q^{2} \varphi(q) \ell} \sum_{r \mid \frac{n}{\ell}} \frac{\mu(r)}{r} \sum_{m \leqslant r \ell} \sum_{s \leqslant q} \sum_{t \leqslant q} \sum_{a \in \mathcal{A}} \sum_{b \in \mathcal{B}} e\left(\left(\frac{m}{r \ell}-\frac{s}{q}\right) a\right) e\left(\left(\frac{m}{r \ell}-\frac{t}{q}\right) b\right) \\
& \times \sum_{\chi \neq \chi^{0}} \bar{\chi}(c) G(s, \chi) G(t, \chi) \\
\ll & \frac{\tau(q)}{q^{3 / 2} \ell} \sum_{r \mid \frac{n}{\ell}} \frac{\mu^{2}(r)}{r} \sum_{m \leqslant r \ell} \sum_{s \leqslant q} \sum_{t \leqslant q}(s, q)^{1 / 2}(t, q)^{1 / 2} \\
& \times \min \left(\# \mathcal{A},\left\|\frac{s}{q}-\frac{m}{r \ell}\right\|^{-1}\right) \cdot \min \left(\# \mathcal{B},\left\|\frac{t}{q}-\frac{m}{r \ell}\right\|^{-1}\right) .
\end{aligned}
$$


By Möbius transform, we have

$$
\sum_{s \leqslant q}(s, q)^{1 / 2} \min \left(\# \mathcal{A},\left\|\frac{s}{q}-\frac{m}{r \ell}\right\|^{-1}\right)=q^{1 / 2} \sum_{d \mid q} d^{-1 / 2} \sum_{\substack{s \leqslant d \\(s, d)=1}} \min \left(\# \mathcal{A},\left\|\frac{s}{d}-\frac{m}{r \ell}\right\|^{-1}\right) .
$$

Observe that $(n, q)=1$; thus, for $\ell \mid n$ and $d \mid q$, we have

$$
\left\|\frac{s}{d}-\frac{m}{r \ell}\right\| \geqslant \frac{1}{d r \ell}
$$

from which and Lemma 5, the left-hand side of (12) is bounded by

$$
\begin{aligned}
q^{1 / 2} & \sum_{d \mid q} d^{-1 / 2} \sum_{\substack{s \leqslant d \\
(s, d)=1}} \min \left(\# \mathcal{A}, d r \ell,\left\|\frac{s}{d}-\frac{m}{r \ell}\right\|^{-1}\right) \\
& \ll q^{1 / 2} \sum_{d \mid q} d^{-1 / 2}(\min (\# \mathcal{A}, d r \ell)+d \log d) \\
& =\# \mathcal{A} q^{1 / 2} \sum_{\substack{d \mid q \\
d>\# \mathcal{A} / r \ell}} d^{-1 / 2}+r \ell q^{1 / 2} \sum_{\substack{d \mid q \\
d \leqslant \# \mathcal{A} / r \ell}} d^{1 / 2}+q^{1 / 2} \sum_{d \mid q} d^{1 / 2} \log d \\
& \ll(r \ell)^{1 / 2}(\# \mathcal{A})^{1 / 2} q^{1 / 2} \tau(q)+q \tau(q) \log q,
\end{aligned}
$$

and similarly

$$
\sum_{t \leqslant q}(t, q)^{1 / 2} \min \left(\# \mathcal{B},\left\|\frac{t}{q}-\frac{m}{r \ell}\right\|^{-1}\right) \ll(r \ell)^{1 / 2}(\# \mathcal{B})^{1 / 2} q^{1 / 2} \tau(q)+q \tau(q) \log q .
$$

Thus,

$$
I_{2} \ll \sqrt{\frac{\# \mathcal{A} \# \mathcal{B}}{q}} \tau^{3}(q) \cdot n 2^{\omega(n / \ell)}+q^{1 / 2} \tau^{3}(q) \log ^{2} q \cdot 2^{\omega(n / \ell)},
$$

where $\omega(n)$ denotes the number of distinct prime factors of $n$.

Combining (8), (9) and (13), we can deduce the theorem immediately.

4. Remarks. Recalling that $\mathcal{Q}$ is a reduced residue system modulo $q$, and taking $q=p$ as a prime number, $\mathcal{A}=\mathcal{B}=\mathcal{Q}, n=2, \ell=1$ in Theorem 1 , we can obtain

$$
N(p)=\frac{1}{2} p+O\left(p^{1 / 2} \log ^{2} p\right)
$$

which is just the same as (1). Similarly, Theorem 1 yields (2) with a slightly weaker error term.

Taking $\mathcal{A}=\left\{n \in \mathcal{Q}: 1 \leqslant n \leqslant \theta_{1} q\right\}, \mathcal{B}=\left\{n \in \mathcal{Q}: 1 \leqslant n \leqslant \theta_{2} q\right\}$,

$$
r_{n}\left(\theta_{1}, \theta_{2}, c ; q\right)=\sum_{\ell \mid n} N(\mathcal{A}, \mathcal{B}, c, n, \ell ; q)-N(\mathcal{A}, \mathcal{B}, c, n, n ; q),
$$


and hence

$$
\begin{aligned}
r_{n}\left(\theta_{1}, \theta_{2}, c ; q\right) & =\sum_{\ell \mid n} \frac{\theta_{1} \theta_{2}}{n} \varphi\left(\frac{n}{\ell}\right) \varphi(q)-\frac{\theta_{1} \theta_{2}}{n} \varphi(q)+\left(q^{1 / 2} \tau^{3}(q) n \tau^{2}(n) \log ^{2} q\right) \\
& =\left(1-\frac{1}{n}\right) \theta_{1} \theta_{2} \varphi(q)+O\left(q^{1 / 2} \tau^{3}(q) n \tau^{2}(n) \log ^{2} q\right),
\end{aligned}
$$

which is slightly better than (3).

Observing that the condition $2 \nmid a+b$ is equivalent to $a+b \equiv 1(\bmod 2)$, thus we can consider another generalization of the D. H. Lehmer problem over short intervals.

Let $q \geqslant 3, \ell \geqslant 1$ be fixed integers, $n$ and $c$ be integers with $(n c, q)=1$. We define

$$
T(\mathcal{A}, \mathcal{B}, c, \ell ; q, n)=\#\{(a, b) \in \mathcal{A} \times \mathcal{B} \mid a b \equiv c(\bmod q), a+b \equiv \ell(\bmod n)\},
$$

where $\mathcal{A}, \mathcal{B}$ are defined as before. Using the same method above, we can also prove that

$$
T(\mathcal{A}, \mathcal{B}, c, \ell ; q, n)=\frac{\# \mathcal{A} \# \mathcal{B}}{n} \varphi^{-1}(q)+O\left(q^{1 / 2} \tau^{3}(q) \log ^{2} q\right),
$$

which also yields (1), (2) and (3).

ACKNOWLEDGEMENTS. The authors would like to express their sincere thanks to the referees for their helpful comments and suggestions.

\section{REFERENCES}

1. T. Estermann, On Kloosterman's sum, Mathematika 8 (1961), 83-86. 2004).

2. R. K. Guy, Unsolved Problems in Number Theory, 3rd. edn (Springer-Verlag, New York,

3. Y. Lu and Y. Yi, On the generalization of the D. H. Lehmer problem, Acta Math. Sinica, English Ser. 9 (2009), 1269-1274.

4. C. D. Pan and C. B. Pan, Goldbach Conjecture (Science Press, Beijing, 1981).

5. I. E. Shparlinski, On a generalised Lehmer problem for arbitrary powers, East-West J. Math. Special Vol. (2008), 197-204.

6. I. E. Shparlinski, On a generalisation of a Lehmer problem, Math. Zeitschrift $\mathbf{2 6 3}$ (2009), 619-631.

7. Y. Yi and W. Zhang, On the generalization of a problem of D. H. Lehmer, Kyushu J. Math. 56 (2002), 235-241. $307-316$.

8. W. Zhang, On D. H. Lehmer problem, Chin. Sci. Bull. 21 (1992), 1765-1769.

9. W. Zhang, A problem of D. H. Lehmer and its generalization, Compos. Math. 86 (1993),

10. W. Zhang, A problem of D. H. Lehmer and its generalization(II), Compos. Math. 91 (1994), 47-56. 